\title{
INVESTIGACIÓN
}

Recibido: 13/05/2016 --- Aceptado: 08/09/2016 --- Publicado: 15/03/2017

\section{LA COMUNICACIÓN PERIODÍSTICA DE LA GASTRONOMÍA EN ESPAÑA EN EL PRIMER TERCIO DEL SIGLO XX (1900-1936)}

$\mathbf{M}^{\mathrm{a}}$ Dolores Fernández Poyatos ${ }^{1}$ : Universidad de Alicante. España dolores.fernandez@ua.es

Ainhoa Aguirregoitia Martínez: Universidad de Alicante. España aam47@alu.ua.es

\section{RESUMEN}

Durante el primer tercio del siglo XX, la gastronomía fue un tema de interés en la prensa española, que recogió abundantes noticias y artículos de notables cocineros como Ignacio Doménech y Teodoro Bardají, pero también de reconocidos periodistas y escritores -Dionisio Pérez, Pardo Bazán, Carmen de Burgos- que reivindicaron la defensa de la cocina nacional, abogaron por mejorar y ampliar la enseñanza culinaria doméstica y profesional y divulgaron asuntos de historia gastronómica e innovaciones y avances técnicos. En el presente trabajo se expone el análisis de la gastronomía en revistas ilustradas, semanario y diarios de la época a través del catálogo online de la Biblioteca Nacional. Los resultados muestran, por una parte, el valor de la prensa como una fuente primordial para conocer la historia de la gastronomía en España en un período poco investigado y, paradójicamente, bastante reprobado; por otra, la abundancia de los documentos hallados, mucho de ellos inéditos, permite confirmar el importante movimiento gastronómico que hubo en España durante esos años.

PALABRAS CLAVE

Gastronomía - Periodismo - Historia - Siglo XX - Culinaria española Comunicación periodística.

\section{JOURNALISTIC COMMUNICATION OF GASTRONOMY IN SPAIN DURING THE FIRST THIRD OF THE TWENTIETH CENTURY (1900- 1936)}

\footnotetext{
$1 \mathrm{M}^{\mathrm{a}}$ Dolores Fernández Poyatos: profesora de la Universidad de Alicante, donde desarrolla su actividad docente e investigadora en diversos grados y másteres. dolores.fernandez@ua.es
} 


\begin{abstract}
During the first third of the twentieth century, gastronomy was a topic of interest in the Spanish press, which compiled news and articles from prominent chefs such as Ignacio Domenech and Teodoro Bardaji, but also from renowned journalists and writers, such as Dionisio Perez Pardo Bazan, Carmen de Burgos, who vindicated the defense of the national cuisine, interceded to improve and expand the domestic and professional culinary education and disseminated issues of gastronomic history and innovations and technical advances. In this paper, the analysis of gastronomy in pictorials, weeklies and newspapers of the time is presented through the online catalogue of the National Library. The results show, on the one hand, the value of the press as a primary source to know the history of gastronomy in Spain in a little researched and, paradoxically, quite reprobate period; on the other hand, the abundance of documents found, many of them unpublished, confirms the important gastronomic movement that took place in Spain during those years.
\end{abstract}

\title{
KEYWORDS
}

Gastronomy -Journalism - History - Twentieth Century - Spanish Culinary Journalistic Communication

\section{A COMUNICAÇÃO PERIODÍSTICA DA GASTRONOMIA NA ESPANHA NO PRIMEIRO TERÇO DO SÉCULO XX(1900-1936).}

\section{RESUMO}

Durante o primeiro terço do século XX, a gastronomia foi um tema de interesse na imprensa espanhola, que dedicou abundantes noticias e artigos de notáveis cozinheiros como Ignácio Doménech e Teodoro Bardají, mas também de reconhecidos jornalistas e escritores- Dionísio Pérez, Pardo Bazán, Carmen de Burgos- que reivindicaram a defesa da cozinha nacional, defenderam por melhorar e ampliar o ensino culinário doméstico e profissional e divulgaram assuntos de história gastronômicas, inovações e avances técnico. No presente trabalho se expõe a analises da gastronomia em revistas ilustradas, semanários e diários da época através do catálogo online da Biblioteca Nacional. Os resultados mostram, por uma parte, o valor da imprensa como uma fonte primordial para conhecer a historia da gastronomia na Espanha em um período pouco investigado e, paradoxalmente, bastante reprovado; por outro lado, a abundância dos documentos encontrados, muitos deles inéditos, permite confirmar o importante movimento gastronômico que houve na Espanha durante esses anos.

\section{PALAVRAS CHAVE}

Gastronomia - Periodismo - História - Século XX - Culinária espanhola Comunicação jornalística. 


\section{Como citar el artículo}

Fernández Poyatos, M ${ }^{\mathrm{a}}$ D.; Aguirregoitia Martínez, A. (2017). La comunicación periodística de la gastronomía en España en el primer tercio del siglo XX (1900-1936) [Journalistic communication of gastronomy in Spain during the first third of the twentieth century (1900-1936)] Vivat Academia. Revista de Comunicación, $\mathrm{n}^{\mathrm{o}}$ 138, 7795. doi: http://dx.doi.org/10.15178/va.2017.138.77-95. Recuperado de http://vivatacademia.net/index.php/vivat/article/view/996

\section{INTRODUCCIÓN}

\subsection{El discurso gastronómico durante el primer tercio del siglo XX}

A lo largo del primer tercio del siglo XX, se observa en la prensa española una progresiva colaboración de personalidades interesadas en el tema gastronómico. Se trataba de célebres cocineros, pero también de periodistas, escritoras y eruditos, amantes de la buena comida, aunque, sobre todo, de la cuestión culinaria en España, participando algunos de ellos con secciones, columnas y noticias de periodicidad fija o esporádica (la mayoría de las veces). Los más relevantes y de mayor participación y continuidad fueron Ignacio Doménech, Teodoro Bardají y Dionisio Pérez. Los dos primeros -cocineros de profesión- están considerados los principales de su época y los precursores de la gastronomía española moderna; ambos publicaron notables libros con recetas, siendo, igualmente, tenaces defensores de nuestra cocina.

Doménech es reconocido como editor de dos revistas de cocina -La Cocina Elegante (1904-1905) y El Gorro Blanco (1906-1945)-. Además, dejó su legado en importantes semanarios entre los que destacan sus contribuciones en La Moda Elegante en 1920 y en La Hormiga de Oro desde 1925 hasta 1932, con una sección llamada "La Manduca", donde decía idear recetas ex profeso para la revista. A partir del 31 de marzo de 1932, y hasta agosto de 1933, contribuyó en la misma revista con la sección "Recetas culinarias", extraídas de su libro La nueva cocina elegante española.

Teodoro Bardají escribió en las cabeceras culinarias más importantes del momento: La Cocina Elegante, El Gorro Blanco -ambas de Doménech-, Paladar, La Confitería Española y Ellas, publicando en la última una sección con numerosas recetas entre 1932 y 1934. Hay evidencia de algún texto crítico como el hallado en el periódico $L a$ Libertad el 10 de enero de 1930, con una reseña a la reedición de El libro de los guisados de Ruperto de Nola, donde criticó el olvido de la cocina española por parte de casi todos los escritores nacionales que, por costumbre, glorificaban a Brillat-Savarin y olvidaban a nuestros escritores del Siglo de Oro. Asimismo, fue uno de los precursores en el uso de otras formas de comunicación como la radio, que comenzaba en el primer tercio del siglo $\mathrm{XX}$ a ser un nuevo y sorprendente medio; hemos constatado su participación durante 1934 en Radio España con "Recetas culinarias", dentro del programa patrocinado por la revista Ellas (23-12-1934, p. 20).

Mención especial requiere la abundante contribución periodística de Dionisio Pérez (Post-Thebussem), quien en 1926, y desde el diario $L a V o z$, se felicitaba de que volviera a estar de moda hablar de culinaria en los periódicos nacionales, tal y como reclamaba en sus escritos el Dr. Thebussem cincuenta años atrás (1888). Durante cuatro años, firmó una columna en la que dejó un estimable testimonio de su ideario culinario junto a recetas, platos y productos de las regiones españolas que, en cierta 
medida, podía ser un reflejo del interés por la identidad regional que desde finales del siglo XIX se extendió por Europa (Storm, 2011, pp. 162-163). Entre la profusión de temas prevalece la defensa de la cocina española y su reivindicación frente a la francesa, a la que estimaba y reconocía por su interés y contribución al arte culinario, pero a la que reprobaba e incluso vituperaba cuando se adueñaba de platos y productos españoles como la salsa mahonesa ( $L a V o z, 18-5-1929$, p. 2); de conglomerado de plagios, copias y raptos, tildó a la cocina gala en otras revistas en que colaboró como Alrededor del Mundo. Allí comenzó en noviembre de 1929 a publicar por entregas amplios extractos de su Guía del buen comer español, editado ese mismo año por el Patronato Nacional de Turismo y que hoy está considerado uno de los grandes tratados de la culinaria nacional.

Las invectivas contra el desprecio y la mala prensa de que es objeto la cocina española no faltaron en sus artículos. Especialmente duro fue el que dirigió a Gregorio Marañón en Nuevo Mundo a propósito de un texto en el que el médico desacreditaba nuestra cocina (24-1-1930, p. 57). A pesar de que empezó a colaborar con el semanario de Perojo a principios de 1929, y a pesar de haber anunciado fija su sección "El fogoncillo nacional de Post-Thebussem", apenas llegó a escribir media docena de artículos en los que aprovechó para censurar las mentiras que desde Francia se vertían con frecuencia sobre nuestra cocina, y para otras cuestiones de cortesía social como la de mostrar su agradecimiento tras ser nombrado Presidente honorario de la Asociación de cocineros de Cataluña y del Sindicato de cocineros de Madrid.

Otros temas que trató versaban sobre recetas y productos como, por ejemplo, arroz, bacalao, fresas, quesos, pimentón, tomate, cerezas, chocolate, bebidas refrescantes para el verano -agua de cebada, hidromel, agua anisada en botijo, guarapo (desterrados ahora por la cerveza helada)-, melones, cebollas, plátano pintón, perdices...; sobre sus beneficios para la salud o sobre cómo aumentar su consumo y producción; sobre costumbres y platos en fiestas renombradas como la cena de Año Viejo de los reyes de España o el roscón de Navidad.

También hizo interesantes propuestas a fin de mejorar la cultura gastronómica del país. Tomando como referencia a Francia, planteó promover ferias regionales y exposiciones internacionales; en particular, las que en Sevilla y Barcelona iban a celebrarse en 1929, para lo que ideó construir un pabellón donde se ensalzara y enseñara la culinaria española; serviría para desterrar la mala y escasa idea que de ella se tenía, para atraer turistas que con el reclamo de las degustaciones permanecerían más tiempo en el país y para que los españoles conociesen mejor su cocina. No en vano, y en síntesis, Dionisio Pérez fue considerado por sus contemporáneos como el digno continuador del Doctor Thebussem (Mundo gráfico, 22-1-1930, p. 3).

Grosso modo, hay que decir que junto a la defensa a ultranza de la cocina española y el rechazo categórico de la francesa -a excepción de Camba que afirmaba que era la mejor y casi única del mundo (2013, p. 46)-, existieron quejas comunes en periódicos y tratados de la época por parte de casi todos los escritores que criticaron el abandono que los españoles tenían de la ciencia y la cocina, así como la inexistencia de una cocina nacional, con la salvedad del cocido -plato por excelencia de nuestra 
culinaria- (Luján y Perucho, 1972, pp. 104-132; Martínez, 1981, p. 401), aunque, eso también, apreciaban ya nuestro rico regionalismo gastronómico: Valencia, Andalucía, Extremadura, Cataluña, País Vasco... conformaban un feraz mapa gastronómicoque, con las debidas atenciones, era capaz de igualarse a los de las naciones más valoradas (Ingram, 2012, p. 78).

Al margen de estos prestigiosos gastrónomos, hubo abundantes evidencias en la prensa de colaboradores (anónimos en su mayoría), cuyo número aumentaba según avanzaba el siglo. Fueron noticias breves, artículos e incluso reportajes extensos que abarcaron un amplio número de temas: recuerdos elogiosos a los grandes maestros franceses -Brillat-Savarin, Carême, Escoffier- y a los notables cocineros y tratadistas clásicos y contemporáneos españoles, reivindicaciones de la cocina nacional, defensa de los productos y elaboraciones propias, su historia, propiedades y usos, preocupaciones relacionadas con la salud que abogaban por una alimentación sana, crónicas de costumbres exóticas y platos remotos -China y Latinoamérica, sobre todoy recetas y reseñas de bibliografía culinaria. Su existencia pone de manifiesto el interés que en nuestro país existía por el arte coquinario. Así pues, la reivindicación del Dr. Thebussem en 1870, y la de contemporáneos y sucesores, de que la cocina tuviera una mayor presencia en los periódicos de la época comenzó a ser una realidad. De su testimonio pretende dar cuenta la presente investigación.

\section{OBJETIVOS Y METODOLOGÍA}

El objetivo del presente trabajo es conocer, a través de la prensa de la época, la gastronomía en España durante el primer tercio del siglo XX. Este propósito tiene su justificación por el desconocimiento que se tiene de un período en el que hubo una copiosa producción editorial, tal y como han puesto de manifiesto investigaciones como la de Simón (2003), quien afirma que las obras publicadas en nuestro país pueden equipararse a las de países líderes como Francia e Italia. En efecto, entre 1900 y 1936 se han constatado doscientos títulos nuevos que, de considerar sus reediciones, llegan a superar los dos mil ejemplares (2003, pp. 274-401). A tenor de estos datos, no es muy descabellado pensar que esta nutrida bibliografía tuvo su correlato en la prensa del momento, donde colaboraron asiduamente importantes personalidades de la época -cocineros, periodistas, escritoras-, tal y como comentamos antes.

Para la recopilación y selección de hechos se han utilizado tres clases de fuentes: bibliografía de época, bibliografía contemporánea y fuentes hemerográficas, siendo las dos últimas consultadas y analizadas a través de las colecciones digitalizadas de portales con acceso libre a sus fondos (Malalana, 2004; Checa, 2008); en particular, los de la Biblioteca Nacional de España (BNE) y la Hemeroteca municipal de Madrid (HMM).

La herramienta metodológica empleada ha seguido la utilizada para un estudio similar por Aguirregoitia y Fernández (2015). La muestra de estudio se ha obtenido aplicando el análisis del discurso a la prensa entre los año 1900 y 1936 del catálogo online de la Biblioteca Nacional a través de las palabras clave gastronomía y culinario(a). Se ha decidido este período de estudio por el grado de madurez que el sector gastronómico alcanzó en este primer tercio del siglo. En total, se han recuperado 3.121 resultados, de los que 2.605 corresponden al término culinario(a) y 
516, a gastronomía. Respecto de los contenidos, se ha considerado oportuno agruparlos en cinco categorías: historia, tecnología e innovación, formación, curiosidades y recetas.

\section{RESULTADOS Y DISCUSIÓN}

\subsection{Historia de la gastronomía}

Las noticias de asunto histórico tienen una presencia discreta en el primer tercio del siglo XX. Hay que resaltar, sin embargo, algunas descripciones del arte culinario como la de la antigua Venecia, que destacaba por el lujo de sus banquetes y la admiración que sentían por los cocineros. Hasta tal punto debió de llegar el fausto que en el siglo XV se dictaron leyes para impedir el lujo desenfrenado en los banquetes. En 1450 se prohibió al anfitrión gastar más de medio ducado para cada uno de sus convidados, y poco después fue prohibida la venta de faisanes, pavos reales, pollos silvestres y truchas. No obstante, el boato continuó, así como la costumbre de "perfumar la comida y rociar ciertos manjares con polvos de oro, que, según general creencia, tenían el don de rejuvenecer el corazón" (El Siglo Futuro, 26-91903, p. 3).

Llaman la atención varios reportajes sobre el arte culinario en China y el exotismo de sus finos manjares entre los que figuran cangrejos con huevos, aletas de tiburón, pechugas de codorniz y "los repugnantes nidos de pájaros, que nosotros llamamos de golondrina" (La Época, 16-7-1900, p. 2). Se trata del denominado "caviar del este" o "alimento medicinal" (Marcone, 2005, p. 1125), carísimo producto considerado una delicadeza culinaria en China y que la prensa española ya había dado a conocer a mediados del siglo XIX. Unas costumbres que se relataban y censuraban eran los platos de carne de perro y gato, aunque en otros reportajes se desmentía su uso ( $L a$ España Moderna, 11-1900, pp. 30-35). No obstante, y a la par que productos apreciados -cangrejos, mejillones, pescados, frutas, dulces-, diversos manjares difíciles de comer en Occidente fueron objeto de atención: orugas saladas, huevos de lagarto, gusanos de tierra, larvas de abejas silvestres en salmuera o fritas... eran exclusivos alimentos para ricos (Alrededor del Mundo, 12-8-1922, p. 17). Como se aprecia, esta búsqueda de lo exótico para causar impacto en el lector conlleva, en ocasiones, el peligro de resaltar lo diferente y olvidar lo más habitual de una cultura alimentaria lejana y desconocida, de forma que se suele producir una falta de visión de conjunto y de rigor científico (Pérez Samper, 2009, p. 109).

Asimismo, se publicaron dilatados reportajes sobre historia de la gastronomía; en general, se retrotraen a Grecia, que empezó a desarrollar el gusto por las buenas comidas en la época de Pericles; con todo, fueron los romanos quienes llevaron a su máxima expresión el lujo a la mesa, el fausto de las salsas, las sabrosas y suculentas carnes y pescados; a ellos debe Europa la aclimatación de sabrosos frutos como la cereza, el albaricoque o la frambuesa. También los árabes fueron maestros en componer comidas. Ahora bien, el arte de comer refinadamente no existió como tal hasta el siglo XVIII en Francia que ostentaba "con orgullo los títulos de maestra e introductora del sibaritismo culinario" (Alrededor del Mundo, 31-8-1905, pp. 11-12). 
Más prolija en detalles es la historia rememorada bajo el epígrafe "La cocina y la mesa" en el Almanaque Bailly-Bailliere de 1934. Si de obligada referencia eran Grecia y Roma, no parecía tan habitual el paso por la Edad Media que el autor nombró, considerando el buen comer en las abadías y los monasterios. Fue en ese período cuando se introdujeron el ajo y el perejil, y la historia culinaria española estuvo representada por una abundante bibliografía: el Arte cisoria del marqués de Villena, los manuscritos de Sancho de Savara (trinchante mayor de Juan II), las recetas de Maese Ivachin (jefe de las cocinas de Fernando el Católico), las de Lopera (confitero de la reina doña Juana) y las de otros maestros que guisaron para próceres y reyes. Fueron los años en que se introdujeron los largos y lujosos banquetes; como ejemplos, las cuarenta y cinco personas -cocineros, reposteros, salseros, panaderos, cerveceros, sirvientes de cava, etc.- que Carlos V llevó a su retiro de Yuste para satisfacer sus deseos culinarios, o los más de quinientos platos con que el conde de Benavente obsequió a Felipe II. Por este tiempo, los nombres de los cocineros iban casi siempre unidos a los de sus señores: Montiño a Felipe IV y Vatel al príncipe Condé en Francia. Acaba la descripción con una breve estampa de los tiempos modernos que vaticinan el fin de los grandes señores, los grandes banquetes y las comidas de duelo; y al contrario, se imponen el sándwich, las empanadas, las tapas, habiéndose apropiado las cocinas de manjares y guisos extranjeros, de frutas tropicales y vinos de todas partes.

La única muestra de la historia gastronómica en España la firma el periodista Pedro de Répide en un suplemento de Blanco y Negro de 1936 ( $\mathrm{n}^{\circ}$ 30, pp. 12-13). Se trata en realidad de un extracto, en su mayor parte literal, de la obra de Dionisio Pérez (1929, pp. 9-39): desde una detenida enumeración de lo más sobresaliente en la Edad Media, a las costumbres de Carlos V con la expansión de la cocina española, recalcando las aportaciones de otras cocinas en nuestra culinaria como el aceite de Roma; el ajo de Egipto o el chocolate de Latinoamérica. Ahora bien, De Répide enriquece su artículo con reseñas de bibliografía culinaria española: Diego Granado y Miguel de Baeza, preceptistas del siglo XVI; Juan de la Mata, con su Arte de repostería y Juan Altamiras del XVIII y el resurgimiento de la gastronomía nacional a finales del siglo XIX con Ignacio $\mathrm{M}^{\mathrm{a}}$ Bueno, el Dr. Thebussem y Ángel Muro. También son interesantes, por parciales e injustas, las apreciaciones negativas sobre Pardo Bazán y Carmen de Burgos y los tratados de cocina que ellas publicaron. Por último, es preciso reparar en la vindicación que De Répide hizo de la cocina española frente a la francesa; así, por ejemplo, es el caso de la olla podrida, las anguilas a la real, las perdices a la Medinacelli, la mahonesa y la llamada con desatino "tortilla a la francesa", cuya receta daba ya en 1637 Montiño, denominándola tortilla a la Cartuja o tortilla doblada.

Novedosa es la columna del escritor Luis Araujo a propósito del nacimiento de la historia de la crítica culinaria en Francia en 1704 con Le Cerf de la Viéville, en cuya obra -Comparación entre la música italiana y la música francesa- se definen los caracteres de una y otra música nacional por las disposiciones gastronómicas de ambos pueblos. Desde entonces hasta sus precursores más actuales -Brillat-Savarin, Grimod de La Reynière, Carlos Monesslet y Berchaud-, esta nueva forma de crítica no ha hecho más que enriquecerse (La Época, 29-7-1922, p. 5). Por último, hay que señalar la interesante historia de los cocineros franceses que publicó Colombine pseudónimo de la escritora y periodista Carmen de Burgos-, donde repasó el 
primordial papel en la evolución de la culinaria que desempeñaron los ilustres Vatel, Tirel, Carême y Brillat-Savarin (Elegancias, 6-6-1925, p. 56).

\subsection{Tecnología e innovación}

Para el progreso culinario es importante que los avances tecnológicos lleguen prácticamente por igual a las distintas clases sociales. De esta forma, si la alta cocina trabaja buscando nuevas técnicas que le permitan moldear productos a su antojo para diseñar un plato final, la cocina popular debe cuidar también dichos avances y aplicarlos siempre que le sea posible a su cocina doméstica, obviando, claro está, el empleo de técnicas imposibles o el uso de productos inaccesibles en el ámbito doméstico. La historia pone de manifiesto la necesidad por parte de cocineros profesionales o no- de conocer y usar inventos y novedades que le ayuden en su quehacer de forma rápida y sencilla.

Entre los agentes que influyen en la historia y evolución gastronómica de un país, la tecnología e innovación son elementos determinantes. Las noticias halladas en la prensa demuestran que la necesidad de una reducción del tiempo empleado en la cocina ya era un quebradero de cabeza para ingenieros y amas de casa, sintiéndose como excesiva y prolija una manipulación que durase más allá de algunos minutos, lo que significó en esta época idear numerosos e ingeniosos utensilios con la finalidad de agilizar el proceso, así como de aliviar la fatiga para el ama de casa y para los cocineros especializados: "limpieza, precisión, prontitud y economía son sus inapreciables ventajas" (Hojas Selectas,1-1906, p. 81).

De las noticias sobre utensilios de aplicación culinaria, destacan por su singularidad y, por qué no, por su similitud con la actual maquinaria doméstica, la batidora, utilizada para batir cremas, huevos, natas y otros ingredientes de repostería fina y la máquina americana para el amasado del pan que permitía elaborarlo "en las mismas casas con mayor facilidad, prontitud y conveniencia que se hace en las de campo" (Hojas Selectas,1-1906, p. 809). En 1930 se publicitaron como novedad la creación de la rejilla de la freidora para retirar los alimentos del aceite hirviendo o la tostadora con forma de sartén que permitía tostar el pan uniformemente y sin quemarse. Además, encontramos utensilios tan sofisticados como el empleado para quitar los huesos de las cerezas, aceitunas y frutas de pequeño tamaño. Estaba claro que la electricidad ofrecía sus ventajas a la culinaria doméstica y profesional, dotándola de más rapidez y menor esfuerzo: “El ideal de la mujer de gobierno y señora de su casa es la eliminación de todo vehículo de suciedad y la supresión, ó por lo menos de la disminución del trabajo manual"; aparecen ahora diferentes máquinas eléctricas; para exprimir frutas, para limpiar cuchillos y tenedores, para triturar carne o para moler café: "Ya ven pues, nuestras laboriosas lectoras cómo la electricidad, agarrada del brazo con la higiene, promete convertir nuestras moradas en pedacitos de cielo" (Hojas Selectas,1-1913, pp. 507-508).

El 9 de agosto de 1911 quedó registrada en Alrededor del Mundo una revolución culinaria: "La cocina al papel" de M. Soyer -químico y cocinero- que, inspirado en las chuletas al papillote, pensó que sería muy ventajoso cocinar todo tipo de manjares en bolsas de papel especiales, impermeables y resistentes a las altas temperaturas. Entre 
la cocina y la ciencia encontramos durante el primer tercio del siglo $\mathrm{XX}$ a personajes como Pozerski de Pomiane, cocinero y científico, estudioso de los procesos de digestión y protagonista de reportajes en la prensa de la época con el titular de “¿Cocineros o fisicoquímicos? La cocina científica" (Caras y Caretas, 17-6-1933, p. 47). $\mathrm{Al}$ respecto, sorprenden las similitudes con el discurso de la actividad gastronómica actual, lo que nos lleva a pensar que la ciencia y la gastronomía se muestran históricamente unidas. En la Fisiología del gusto de Brillat-Savarin ya se hacía mención a la relación de la gastronomía con la ciencia, indicando la importancia de las diversas descomposiciones que padecían los alimentos al ser cocinados. La ciencia ayuda a asimilar el proceso culinario, no lo entorpece ni complica, es, stricto sensu, explicativo; la gastronomía, nadie lo discute, ha evolucionado a la par que la ciencia y la tecnología.

\subsection{Formación}

Durante el primer tercio del siglo XX, la educación culinaria española se dirigió a la mujer; en las escuelas, cátedras e institutos gastronómicos se inscribieron señoras y señoritas de elevada posición social, cuyo objetivo principal era lograr el dominio del arte culinario, pues en sus manos se hallaba la tarea de alimentar a la familia (Pérez Samper, 2012, p. 28; Pérez Samper, 1997, p. 122). Bien claro lo decía la Estampa: "nada hay como un estómago agradecido. Las casadas lo saben bien; las solteras, tan perspicaces siempre saben que eso es un encanto más" (14-1-1933, p. 5). En 1943, María Mestayer de Echagüe, la Marquesa de Parabere -gastrónoma, historiadora y cocinera de referencia-, confesaba no saber freír un huevo y cuando "vio que su esposo ponía toda clase de pretextos para no volver a casa al mediodía, quedándose a comer en el club, [...] el instinto de preservar su matrimonio, [...] la llevó a interesarse por los temas culinarios" (Echagüen y Echagüen, 2013, p. 18). La formación de estas mujeres se llevó a cabo en centros como la Academia de Cocina de San Sebastián, fundada en 1901 por Félix Ibarguren y Loreto Capella, exjefe este último de las cocinas de Alfonso XIII: "Las clases dedicadas al arte culinario que en San Sebastián existen, sería muy conveniente, para la moderna educación de la mujer, que se crearan en aquellos centros de instrucción donde con escaso gasto pudieran implantarse" (Nuevo Mundo, 5-3-1902, p. 16). 
Figura 1. Academia gastronómica de Madrid.

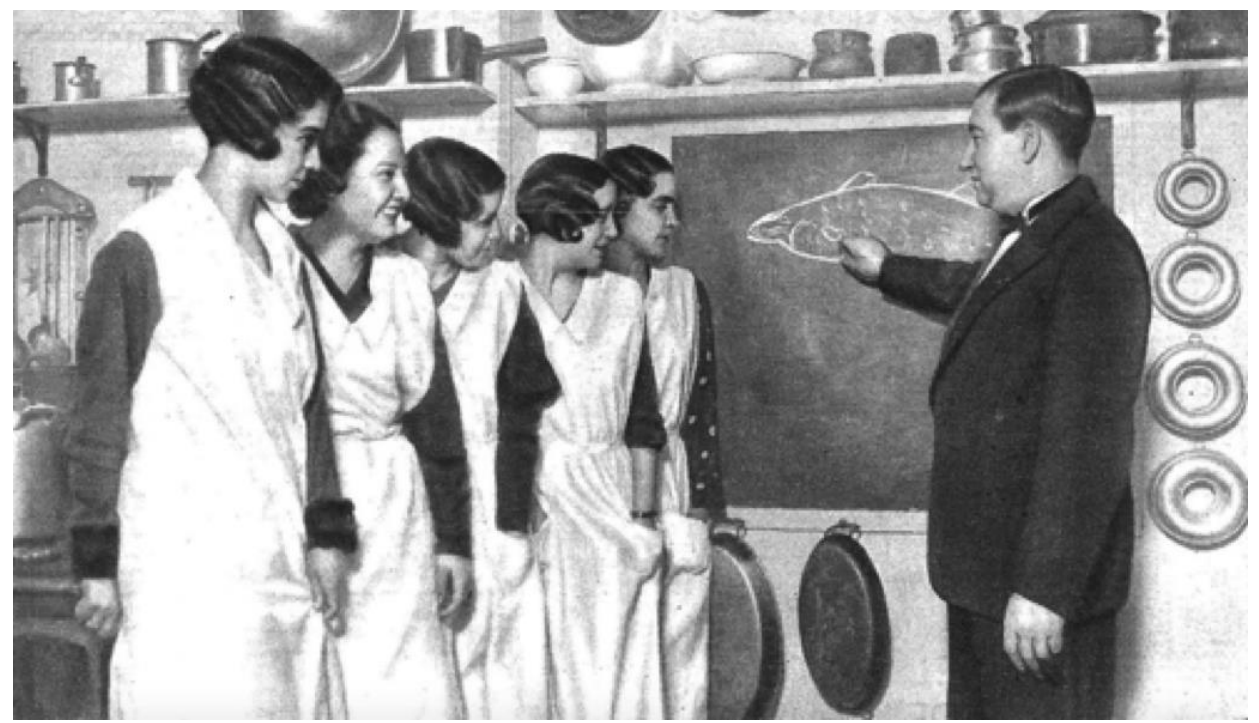

Fuente: Estampa, 14-1-1933, p. 71.

De El Imparcial proviene la noticia de la inauguración de unas clases de cocina para veintidós alumnos, subrayando que el arte culinario está abandonado en España. Entre sus cocineros figuraba Santos Iribarren, estando la teoría a cargo de Ramiro Suárez y siendo una de sus alumnas la celebérrima escritora Pardo Bazán (4 mayo 1905: 3).

Barcelona fue una de las ciudades donde primero se impartieron clases de cocina. Estuvieron a cargo de Ignacio Doménech que creó la primera Escuela española de hostelería en 1908 (Simón, 2012, p. 18), publicando un año después el Reglamento de la Escuela Culinaria y Programa de asignaturas de cocina y el de los Cursos especiales para señoras y señoritas. En La Vanguardia (7-4-1923, p. 5) quedó constancia de que Doménech dio lecciones de cocina práctica familiar en el Centro social de Belén, que fue creado por su hija Nieves (Simón, 2012, p. 4).

En Madrid, se abrió en 1911 la Escuela de Hogar y Profesional de la Mujer; de acceso gratuito, destacó por tener entre sus docentes y administrativos a mujeres, entre las que sobresale Melchora Herrero y Ayora, prestigiosa escritora y autora de tratados de cocina muy populares: El arte de la cocina: fórmulas experimentadas en la Escuela del Hogar (1914) y la Cocina selecta y clásica (1927); escrito el primero en colaboración con su hermana Florencia, quien fue docente en la misma escuela.

También en Madrid impartió clases Doménech cuando se creó en 1920 la primera Academia de Cocina. Fueron conferencias dirigidas a mujeres jóvenes, destinadas con el tiempo a ser madres de familia y directoras de hogares (Simón, 2012, p. 18).

Una de las instituciones más famosas fue la Academia gastronómica de Madrid, creada en 1933 para enseñar a guisar a las señoritas (figura 1). Sus fundadores hicieron hincapié en el empeño por dotar a estos estudios de un halo científico y universitario: "Estamos en una academia gastronómica, universidad donde se estudia la ciencia del hogar, donde se cultiva un arte: el culinario" (Estampa, 14-11933, p. 4). Además de esto, había un interés por popularizar estas escuelas 
haciéndolas accesibles a una clase social media. A pesar de la relevante contribución de las mujeres docentes, el mayor y más importante peso de la formación recayó en maestros varones. Al margen del conocido carácter sexista de la época, existió un nivel de cocina doméstica bastante elevado respecto a otros periodos de la historia, donde las amas de casa, muchas de ellas instruidas en el arte culinario, elaboraban recetas que hoy día por falta de tiempo y práctica no tendrían, quizá, un resultado satisfactorio. La consulta de los recetarios de la época, parcos en datos concretos y ausentes de refuerzo fotográfico, esclarecen nuestro parecer de que el conocimiento culinario de antaño era mejor de lo que se afirmaba: recetas breves, referencias continuas a la intuición de la cocinera -"cuando crea"- o expresiones de medidas inexactas -"con el agua que se considere suficiente". Al respecto, es necesario considerar la opinión de escritores y gastrónomos que calificaban de pobres e inexactos los recetarios de aquellos años; no en vano, en el prólogo de su libro, Melchora y Florencia Herrero reconocían haber redactado las recetas "no à la manera usual de casi todos los libros de esta índole, sino poniendo al principio de cada una la lista de los componentes necesarios y determinando las cantidades en gramos, litros y volúmenes conocidos" (Herrero y Herrero, 1914, p. 8).

Por último, y en lo referente a las noticias internacionales relacionadas con la formación culinaria, hay que destacar en otros países el carácter mixto del alumnado y la profesionalidad de sus objetivos. Mientras España se preocupaba por tener buenas cocineras en las casas, en algunos países europeos se formaban profesionales para el sector. Según Alrededor del Mundo (15-8-1902, p. 108), en Alemania y Austria había "150 escuelas para cocineros. La enseñanza en el arte culinario dura cuatro años, al cabo de los cuales el estudiante recibe un título oficial. Casi todos los jefes de cocina de aquellos países proceden de estas escuelas". En 1930, Berlín ya contaba con escuelas infantiles mixtas que enseñaban ciencias básicas como las matemáticas, la historia y ocupaciones domésticas fundamentales como cocinar, sin distinción de sexos, eso sí.

\subsection{Recetas}

Además de los numerosos recetarios editados en estos años, las recetas llegaron a un gran número de lectores a través de la prensa, sobre todo de revistas para la mujer, pero también por las publicaciones generalistas y más afamadas de la época, así como por las revistas especializadas del sector.

La Última Moda, una de las más longevas y valiosas revistas femeninas, inició en 1903 en su sección Vida práctica un apartado denominado el "Arte culinario", explicando el rechazo que le producía la habitual edición de recetas imposibles en tratados y en la prensa y abogando, en consecuencia, por las recetas sencillas, de fácil manufactura y privativas de la cocina y repostería española (18-1-1903, p. 12). La monárquica y conservadora Ellas mantuvo durante cuatro años un apartado de recetas titulado "La cocina" (1932-1936), en el que, como vimos, participó Bardají.

Otra revista que contó con reputados cocineros fue La Moda Elegante que, a partir de 1920, sintió

la necesidad de dedicar una sección especial a la Cocina, en la que con toda amplitud se diera a conocer de un modo claro y sencillo, no exento de 
amenidad, la confección de los platos más modernos y más antiguos y otras particularidades del arte gastronómico, en forma práctica para nuestras abonadas. [...] Para llevar a cabo nuestro propósito era preciso encomendar esta sección a un gran cocinero, que a la par fuera escritor excelente, [...] nos referimos al autor de La Guía del Gastrónomo y maître d'hòtel, D. Ignacio Domenech (6-1-1920, p. 12).

Una de las publicaciones más destacadas de la época fue el Almanaque Bailly-Bailliére, una pequeña enciclopedia popular de la vida práctica como el mismo subtítulo rezaba. Entre sus numerosos apartados mantuvo "La cocina en casa" durante el primer tercio del siglo, donde publicó recetas de los platos indicados en las minutas mensuales que extraía de su Agenda culinaria y donde incluía información sobre productos de temporada -carnes, pescados, mariscos, legumbres y frutas- en la sección llamada "Calendario gastronómico".

Otras notables revistas incorporaron secciones de recetas en sus números. Nuevo Mundo tuvo "Arte culinario" desde 1903; Alrededor del Mundo, "Recetas y recreos" un año más tarde; Por esos mundos inauguró en 1911 su sección "Páginas femeninas" con un apartado de arte culinario; La Unión Ilustrada lo hizo en 1923, el mismo año que comenzaba La Hormiga de Oro con Doménech. Incluso algunos como Mundial Magazine recibieron solicitudes de sus suscriptoras para que editara en su suplemento quincenal Elegancias un apartado práctico de recetas y principios del arte culinario, como así hizo en 1913.

Lo más apreciable, sin embargo, fueron las revistas del sector, entre las que se distinguen La Cocina Elegante (1904-05) y El Gorro Blanco (1906-1945), ambas fundadas por Ignacio Doménech y de las que la segunda fue quizás la más importante de su época. En ella podemos encontrar apartados que tratan de historia de la gastronomía y sus productos, recetas, repostería, menús de cenas o comidas célebres, curiosidades, teoría y testimonios bibliográficos como la reproducción del intercambio epistolar entre el Dr. Thebussem y Un cocinero de S.M. en La Ilustración Española y Americana (1876 y 1877), y que constituye uno de los debates más rigurosos sobre el estado la culinaria nacional (Anderson, 2014, p. 401).

De similar factura fue la Cocina artística y casera; dirigida por Pascual La Rosa, buscó dignificar la cocina y a los profesionales españoles. Su contenido culinario, entre los que se hallan recetas, quiso ser de interés para la aristocracia, las clases medias y hasta para las más modestas. Como curiosidad, llama la atención su apartado "Cuentos de cocina", donde se narran historias de ficción que siempre discurren en torno a la cocina o a la elaboración de un menú.

Por último, destaca Paladar; probablemente, la revista más tardía del período analizado (1933-1935), y en la que participaron ilustres cocineros y gastrónomos como Gonzalo Avello, Pedro Chicote, Doménech y Bardají. Tuvo un apartado para recetas, bebidas y temas variados de los que sobresale por su novedad y modernidad la sección "Gastrotecnia", donde se explicaban secretos técnicos y se revelaban trucos a fin de mejorar resultados en la cocina. 


\subsection{Curiosidades}

A este grupo pertenecen noticias y reportajes de contenido diverso, cuyo nexo radica en su carácter sorprendente e insólito. Son, en general, testimonios aislados que por sí solos carecen de entidad para constituir una tipología rigurosa. Así, por ejemplo, la creación de la "Sociedad de los 100 kilos" en Francia, en la que solo se ingresaba si se superaba dicho peso y cuyo fin era cultivar la afición a la buena mesa (Alrededor del Mundo, 11-9-1917, p. 7).

A modo de notas de sociedad, mencionar la noticia en $L a V o z$ sobre la boda de un millonario norteamericano con la cocinera de un hotel porque "ésta guisaba muy bien" (1-8-1925, p. 1), o la curiosidad de saber cuáles eran los platos preferidos por célebres personajes como el emperador Augusto, aficionado a los peces pequeños, queso e higos; Enrique IV, rey de Francia, a las ostras y los melones; el filósofo Kant, al puré de lentejas preparado con tocino; lord Byron, al queso añejo de Cheshire y a los pepinos y berzas empapados en vinagre (Caras y Caretas, 14-1-1922, p. 84). Sorprendente era conocer qué cantidad de alimentos consumía Madrid al día o cuánta comida transportaba un trasatlántico: 18.136 kilos de harina; 6.600 ostras; 7.700 kilos de fiambre, 165 de ostras y 21.415 de carne; 17 toneladas de patatas, 45 de hielo; 21.000 limones y 35.000 naranjas; tampoco podía faltar el vino: 21.250 botellas entre Burdeos, Borgoñas, Oportos, vino de Málaga... (Alrededor del Mundo, 26-121901, pp. 11-12).

Otros asuntos provocarían cierta hilaridad en el lector actual. Véase, si no, el sombrero confeccionado con colas de bacalao (Alrededor del Mundo, 21-11-1921, p. 4) o el diseño de un restaurante flotante en la playa de Coney Island (figura 2) para "socorrer a los bañistas que en medio del agua se sientan acometidos de las cosquillas del apetito, para lo cual ha inventado un chaleco salvavidas dispuesto de manera que en él pueda sujetarse una mesa con todos los cachivaches y viandas necesarios para hacer un almuerzo a flote" (Alrededor del Mundo, 6-6-1921, p. 11).

Figura 2. Restaurante flotante

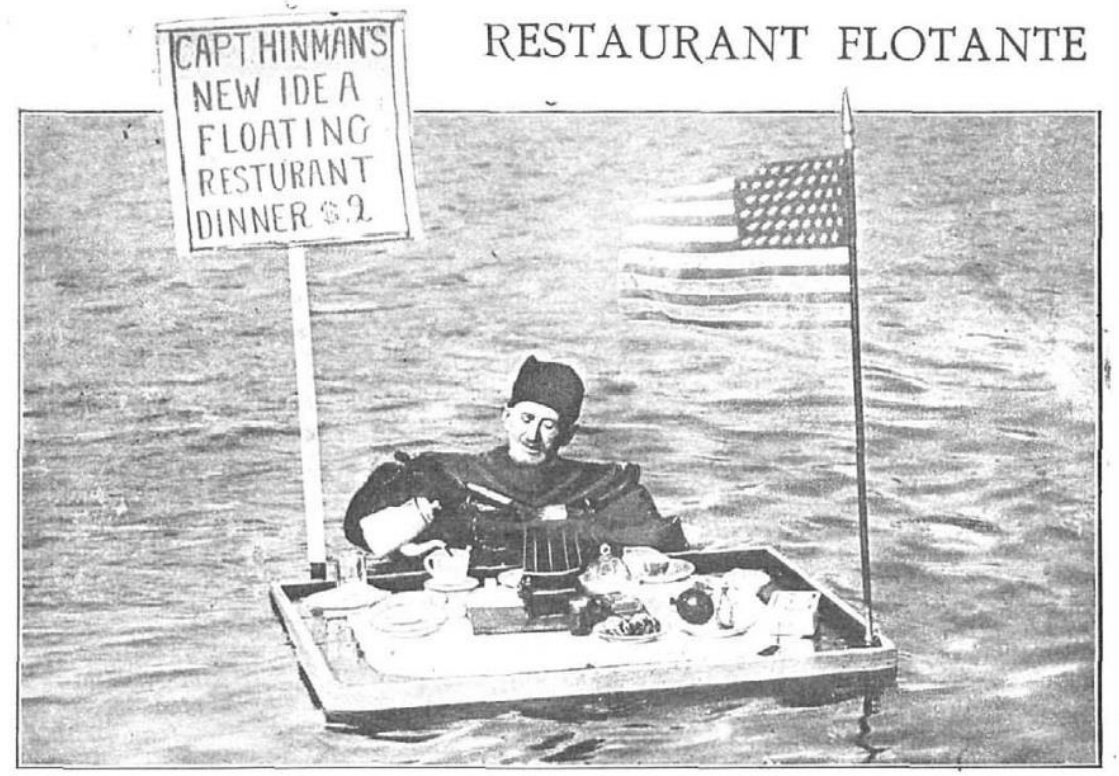

Fuente: Alrededor del Mundo, 6-6-1921, p. 11. 
Bastante menos jocoso es el asunto del envenenamiento que Morató Ventura denunció en las casas de comidas económicas en Madrid por el uso fraudulento de productos, la adulteración de alimentos y el empleo de falsos y nocivos condimentos. Así, por ejemplo, la reutilización de restos de comida para elaborar sopas, purés y consomés o la adulteración con harinas, leche o anilina vegetal para una tortilla ( $\mathrm{La}$ Acción, 30-8-1923, p. 3).

No podían faltar los artículos sobre productos y alimentos insólitos. El cultivo de las praderas de mar -las algas- ya se sugería como una solución para paliar el hambre en épocas de hambruna. No en vano, se conocía su uso en Japón y China (Madrid Científico, 1909, pp. 212-213). Como sabemos, bien por mero gusto, bien por sus virtudes nutricionales, su consumo se ha incrementado notablemente en países occidentales, ocupando un importante lugar en la cocina actual.

También se publicaron propuestas para aprender a alimentarnos con insectos. En un amplio reportaje, se repasan platos a base de insectos a lo largo de la historia y la geografía mundial: las apreciadas langostas en Marruecos y Palestina; el gusto por las cigarras y escarabajos de los antiguos griegos y romanos; las exquisitas hormigas blancas, de miel y larvas-, para africanos e indios de América (Alrededor del Mundo,145-1919, p. 6). Todo ello justificado desde la cultura, ya que, según el autor, todo es cuestión de costumbres o ¿acaso no es más repulsivo un caracol o una ostra? La alimentación y el patrimonio gastronómico de un país es un hecho cultural y, como tal, no es cuestionable por su estética o por desconocimiento (Flandrin, 1987, p. 4). Rigurosas y exhaustivas investigaciones de la comida prehispánica en México están recuperando el valor nutritivo de los insectos que, además, está siendo revalorizado desde la práctica culinaria con la oferta en restaurantes exclusivos y de lujo de sabrosos platos de escamoles, chapulines o gusanos de maguey (Viesca y Romero, 2009).

Acaso, lo más interesante desde la comunicación se halle en el uso de fotografías para explicar la elaboración de recetas, lo que obedecía a las nuevas tendencias en el diseño de la prensa que llegaba de EE.UU. Los periódicos comenzaron en los años veinte y treinta del siglo XX a ser mapas visuales en aras de la legibilidad (Díaz Noci, 1998, p. 2); de ello, no escapó tampoco el periodismo culinario. Se observa por primera vez, y casi única, en La Última Moda, cuando a principios de 1903 anunció el inicio de una nueva sección titulada "Arte culinario", firmada por Cordón Bleu. Durante ese año y el siguiente, publicó recetas acompañadas de fotografías en las que una cocinera mostraba cómo prepararlas (figura 3). 
Figura 3. Primera fotorreceta en la que una cocinera enseñaba a preparar el plumpudding inglés.

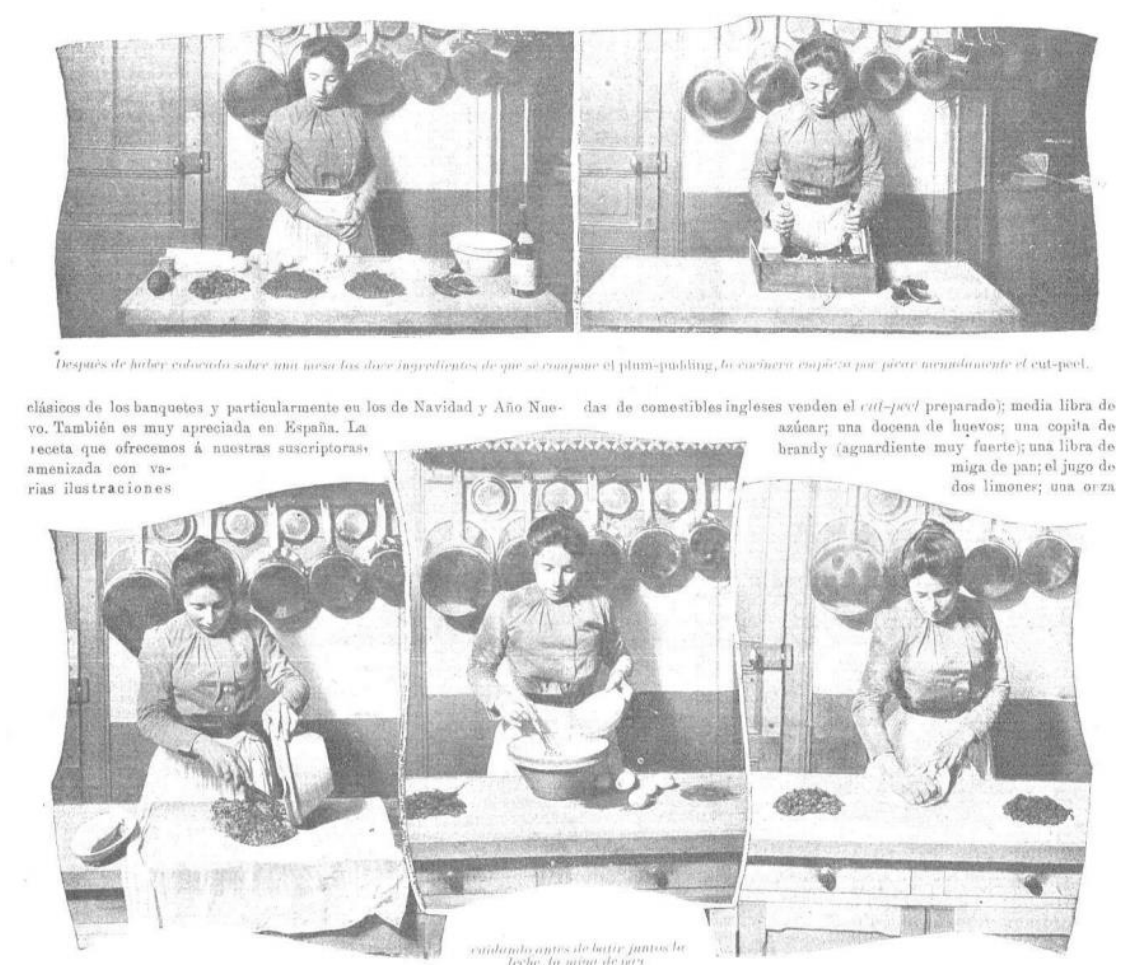

Fuente: La Última Moda, 15-2-1903, p. 13.

Este modelo mostrativo no fue seguido por el resto de la prensa, aunque algunas revistas como Alrededor del Mundo incluyeran, en ocasiones, ilustraciones bastante afortunadas sobre el modo de preparar un pavo para asarlo.

Por último, y desde la comunicación, es preciso señalar la noticia recogida en $E l$ Heraldo de Madrid (1-8-1930, p. 14) sobre la edición en Francia de películas al servicio de su propaganda culinaria; en ellas, se enseñaban las ventajas de la cocina francesa; al parecer, tenían la intención de traducirlas a numerosos idiomas y distribuirlas por bastantes países.

Como última curiosidad destacamos el artículo la "Cocina del amor", donde se ofrecía un menú con productos variados y exquisitos en el que sobresalían las ostras al champagne, las trufas a la veneciana y el consomé de pescado. Según BrillatSavarin "la trufa debe su fama no sólo a su delicioso sabor, sino también a su virtud para sostener y fortalecer facultades cuyo ejercicio lleva aparejados los más dulces goces" (Vida Galante, 22-9-1905, p. 17). No es la única referencia al disfrute carnal; así por ejemplo, de la alcachofa llegó a escribirse: "no puedo comer una alcachofa sin pensar en aquellos caballeros andantes que soñaban con la dama de sus pensamientos, bajo la visera impenetrable, y que una vez terminada justa, al despojarse del casco, recordaba su figura natural de amante tímido" (Mundo Gráfico, 24-9-1930, p. 47). 


\section{CONCLUSIONES}

Tras el estudio, es posible confirmar una presencia relevante de la gastronomía en la prensa española del primer tercio del siglo XX. El análisis llevado a cabo no sólo permite constatarlo, sino también verificar la diversidad de los temas tratados $\mathrm{y}$, sobre todo, el prestigio de los autores que intervinieron: no en vano, numerosos artículos y reseñas fueron rubricados por los cocineros y los gastrónomos más destacados de la época como Ignacio Doménech, Teodoro Bardají, Melchora Herrero y Dionisio Pérez, entre otros.

Uno de los asuntos con mayor presencia en las noticias y artículos de la prensa es el sentir de los gastrónomos que critican el olvido de la cocina española en la producción editorial de la época, así como la vindicación de la cocina nacional. Se censuraba la extraña necesidad de realzar lo extranjero, generalmente lo francés, frente a lo nacional. Estas voces hacían hincapié en la defensa de lo tradicional, abogando por conservar los valores que nos hacen únicos y que forman parte de nuestra idiosincrasia gastronómica: "es cierto que hay que saber guisar á la francesa, á la italiana y hasta á la rusa y á la china, pero la base de nuestra mesa, por ley natural, tiene que reincidir en lo español" (Pardo Bazán, 1913, p. 6). En estos testimonios, sobresalen algunas propuestas (si bien, aisladas) como las de Dionisio Pérez de mejorar la cultura gastronómica mediante la organización de ferias regionales y exposiciones con la finalidad de atraer a turistas e interesados.

La historia de la gastronomía mantuvo tuvo su lugar en los periódicos con narraciones de pantagruélicos banquetes de antaño y con novedosas referencias históricas de la culinaria nacional en aras, muchas veces, de reivindicar nuestros productos, nuestros platos y nuestros cocineros y preceptistas.

En cuanto a la tecnología e innovación, se asume que han estado, y están, siempre presentes, en el desarrollo gastronómico de un país, siendo indisolubles la una de la otra: la gastronomía evoluciona hacia nuevos métodos siempre que la tecnología se lo permita, siendo la parte científica la más racional y técnica, ayudando así al entendimiento de la materia y de las pautas a seguir para una finalidad concreta. Y lo que quizá sea más importante: la necesidad de que el avance tecnológico llegue a todas las clases sociales

Por otra parte, la formación nos muestra el carácter restringido y doméstico de la enseñanza de la época. Dirigida a las mujeres para que hicieran feliz a sus familias, son numerosos los artículos que las animan para una correcta educación en la cocina; y, al contrario, destacan los referidos a la enseñanza en otros países, donde se existía una formación mixta y profesional. No es, pues, extraño el menosprecio del que fueron objeto las que se interesaron por la gastronomía como Pardo Bazán, criticada por Doménech y Bardají o el olvido en el que cayeron otras como Carmen de Burgos, Melchora Herrero o la recientemente recuperada Marquesa de Parabere

En cuanto a las recetas, los tratados fueron el medio por excelencia para divulgarlas; no obstante, la prensa -popular y del sector- mantuvo desde los primeros años del siglo una presencia cada vez mayor. Tanto los semanarios femeninos, como las revistas ilustradas de gran notoriedad, incluyeron en sus números secciones fijas de 
recetas a cargo de prestigiosos cocineros -Doménech, Bardají- o de reconocidos gastrónomos como Dionisio Pérez quienes defendieron las recetas españolas, las de elaboración sencilla y las explicaciones correctas en contraposición a las imprecisiones que abundaban en muchos recetarios y en épocas pasadas.

Respecto al grupo curiosidades (quisquicosas las llamaba Doménech), son abundantes las noticias y reportajes de alimentos, productos y hechos insólitos y curiosos. Desde notas de sociedad de único valor anecdótico, a inventos extravagantes como el sombrero de colas de bacalao. Por el contrario, sí destaca la introducción de la fotografía en los reportajes para elaborar recetas; modelo, como señalamos, que tuvo escaso uso en las revistas, quizá por el elevado coste que ello suponía.

El interés que la prensa comenzó a demostrar por la cocina en el siglo anterior, donde el intercambio epistolar del Dr. Thebussem y Un cocinero de S.M. fue probablemente lo más destacado (Bueno y Ortega, 1998, pp. 1-17), se afianzó en el primer tercio del siglo XX. La sociedad española, al menos aquella de economía desahogada, se interesó por la gastronomía, y si los numerosos recetarios fueron testimonio de ello, también lo fueron las abundantes noticias y reportajes que se publicaron. Su investigación y análisis nos ha permitido ampliar su conocimiento, así como la conveniencia de abundar en ello y de poder afirmar el reconocido valor que la prensa tiene como fuente para la historia de la gastronomía.

\section{REFERENCIAS}

Aguirregoitia, A. \& Fernández, Mª D. (2015). La gastronomía en la prensa española del siglo XIX, Estudios sobre el Mensaje Periodístico, 21, (1), 17-33. Recuperado de: https://revistas.ucm.es/index.php/ESMP/article/view/49078

Anderson, L. (2014). Theunity and diversity of La olla podrida: anautochthonousmodel of Spanish culinary nationalism, Journal of Spanish Cultural Studies, $\mathrm{n}^{\circ} 14,400-414$.

Bueno, P. \& Ortega, R. (1998). De la fonda nueva a la nueva cocina. La evolución del gusto culinario en España durante los siglos XIX y XX, Revista de libros de la Fundación Cajamadrid, 1-17. Recuperado de: http://www.revistadelibros.com/articulos/evolucion-de-la-cocina-espanoladurante-los-siglos-xix-y-xx.

Camba, J. (2013). La casa de Lúculo o el Arte de comer (Nueva fisiología del gusto). Madrid: Catálogo de publicaciones del Ministerio de Educación, Cultura y Deporte.

Checa, A. (2008). La historia de la comunicación y las nuevas tecnologías, en E. Feliu, (coord.). Comunicación, memoria, historia, modelos. Madrid: Edipo.

Díaz Noci, J. (1998). Nacimiento y recepción del diseño periodístico moderno. Las publicaciones en lengua vasca (1921-1936), Zer. Revista de Estudios de Comunicación, 3, 279-295. Recuperado de: http://www.ehu.eus/zer/hemeroteca/pdfs/zer04-13diaz.pdf 
Fernández Poyatos, Ma D.; Aguirregoitia Martínez, A. La comunicación periodística de la gastronomía en España en el primer tercio del siglo XX (1900-1936)

Echagüen, J. A. \& Echagüen, G. (2013). Historia de la gastronomía (Esbozos). Madrid: Real del Catorce editores.

Flandrin, J-L. (1987). Historia de la alimentación: Por una ampliación de las perspectivas, Manuscrits, $\mathrm{n}^{\circ}$ 6, 7-30.

Herrero, M. \& Herrero, F. (1914). El arte de la cocina [Texto impreso]: fórmulas experimentadas en la Escuela del Hogar de Madrid. Madrid: Suc. De Hernando.

Ingram, R. (2012). Mapping and Mocking: SpanishCuisine and Ramon Gómez de la Serna's ‘El primer mapa gastronómico de España', Cincinnati Romance Review, $\mathrm{n}^{\circ}$ 33, 78-97. Recuperado de: http://www.cromrev.com/volumes/vol33/04-vol33Ingram.pdf

Luján, N. \& Perucho, J. (1972). El libro de la cocina española: gastronomía e historia. Barcelona: Danae.

Malalana, A. (2004). Fuentes de información para el análisis de la Publicidad. Las bases de datos publicitarias e Internet, Cuadernos de Documentación Multimedia, $\mathrm{n}^{\circ}$ 15. Recuperado de: $\underline{\text { http://dspace.ceu.es/bitstream/10637/1429/1/2004_15.pdf. }}$.

Marcone, M. F. (2005). Characterization of theediblebird'snestthe Caviar of the East Food, Research International, $\mathrm{n}^{\mathrm{o}}$ 38, 1125-1134. Recuperado de: http://libra.msra.cn/Publication/40902838/characterization-of-the-edible-bird-snest-the-caviar-of-the-east.

Martínez Llopis, M. (1981). Historia de la gastronomía española. Madrid: Editor Nacional.

Pardo Bazán, E. (1913). La cocina española antigua [Texto impreso]. Madrid: Renacimiento.

Pérez, D. (1929). Guía del buen comer español: inventario y loa de la cocina clásica de España y sus regiones. Madrid: Patronato Nacional de Turismo.

Pérez Samper, $\mathrm{M}^{\mathrm{a}}$ de los Á. (1997). Los recetarios de mujeres y para mujeres. Sobre la conservación y transmisión de los saberes domésticos en la época moderna, Cuadernos de Historia Moderna, 19, 121-154. Recuperado de: https://www.ucm.es/data/cont/docs/995-2015-04-06-1997Cuadernos.PDF.

Pérez Samper, $\mathrm{M}^{\mathrm{a}}$ de los Á. (2009). La Historia de la Historia de la alimentación, en Chronica Nova, $\mathrm{n}^{\mathrm{o}}$ 35, 105-162. Recuperado de: http://revistaseug.ugr.es/index.php/cnova/article/view/1632. 
Pérez Samper, $\mathrm{M}^{\mathrm{a}}$ de los Á. (2012). Recetarios manuscritos de la España moderna, Cincinnati Romance Review, $\mathrm{n}^{\mathrm{o}}$ 33, 27-58. Recuperado de: http://www.cromrev.com/volumes/vol33/02-vol33-PerezSamper.pdf.

Simón, Ma del C. (2003). Bibliografía de la gastronomía y de la alimentación en España. Gijón: Ediciones Trea.

Simón, $\mathrm{M}^{\mathrm{a}}$ del C. (2012). Ignacio Doménech. Autor y editor culinario, Cincinnati Romance Review, $\mathrm{n}^{\mathrm{o}}$ 33, 4-26. Recuperado de: http://www.cromrev.com/volumes/vol33/01-vol33-SimonPalmer.pdf.

Storm, E. (2011). La cultura regionalista en España, Francia y Alemania, Ayer, no 82, 161-185. Recuperado de: https://openaccess.leidenuniv.nl/bitstream/handle/1887/19174/cultura\%20regi onalist $\% 20$ AYER.pdf?sequence $=1$

Thebussem, Dr. (1888). La mesa moderna cartas sobre el comedor y la cocina cambiadas entre el Doctor Thebussem y Un cocinero de S.M. Madrid: Librerías de Fernando Fe y Leocadio López y sus corresponsales.

Viesca, F. C. \& Romero, A. (2009). La entomofagia en México. El Periplo sustentable, $\mathrm{n}^{\mathrm{o}}$ 16. $57-83$.

\section{AUTORAS \\ $M^{a}$ Dolores Fernández Poyatos}

Es profesora de la Universidad de Alicante, donde desarrolla su actividad docente e investigadora en diversos grados y másteres: Publicidad y Relaciones Públicas, Turismo y TADE (Turismo y Administración y Dirección de empresas) y máster de Comunicación en Industrias creativas y de Nutrición comunitaria. Ha participado como directora y como investigadora en proyectos de investigación de ámbito internacional, nacional, comunitario y local relacionados con la comunicación. Sus líneas de investigación se centran en la historia de la comunicación, la comunicación turística y en el ámbito de las estrategias comunicativas institucionales. Es autora y coautora de artículos en publicaciones indexadas en bases de reconocido prestigio, así como de comunicaciones a congresos de carácter internacional y nacional.

http://orcid.org/0000-0002-5771-3306

\section{Ainhoa Aguirregoitia Martínez}

Es Licenciada en Publicidad y Relaciones Públicas por la Universidad de Alicante. Con un máster en Dirección y Planificación Turística, se especializa a través de una beca como colaboradora docente en comunicación y estrategia. En la actualidad es doctoranda en la Universidad de Alicante, centrándose sus líneas de investigación en gastronomía, comunicación e historia. Es autora de artículos y comunicaciones a congresos en el ámbito de la comunicación de carácter nacional e internacional. http://orcid.org/0000-0003-3392-3656 